\title{
Hydrogen absorption properties of metal-ethylene complexes
}

\author{
W. Zhou, ${ }^{1,2}$ T. Yildirim, ${ }^{1,2, *}$ E. Durgun, ${ }^{3,4}$ and S. Ciraci ${ }^{3,4}$ \\ ${ }^{1}$ NIST Center for Neutron Research, National Institute of Standards and Technology, Gaithersburg, Maryland 20899, USA \\ ${ }^{2}$ Department of Materials Science and Engineering, University of Pennsylvania, Philadelphia, Pennsylvania 19104, USA \\ ${ }^{3}$ Department of Physics, Bilkent University, Ankara 06800, Turkey \\ ${ }^{4}$ UNAM-National Nanotechnology Research Center, Bilkent University, Ankara 06800, Turkey
}

(Received 23 January 2007; revised manuscript received 6 March 2007; published 30 August 2007)

\begin{abstract}
Recently, we have predicted [Phys. Rev. Lett. 97, 226102 (2006)] that a single ethylene molecule can form stable complexes with light transition metals (TMs) such as Ti and the resulting $\mathrm{TM}_{n}$-ethylene complex can absorb up to $\sim 12$ and 14 wt $\%$ hydrogen for $n=1$ and 2, respectively. Here we extend this study to include a large number of other metals and different isomeric structures. We obtained interesting results for light metals such as $\mathrm{Li}$. The ethylene molecule is able to complex with two $\mathrm{Li}$ atoms with a binding energy of $0.7 \mathrm{eV} / \mathrm{Li}$ which then binds up to two $\mathrm{H}_{2}$ molecules per $\mathrm{Li}$ with a binding energy of $0.24 \mathrm{eV} / \mathrm{H}_{2}$ and absorption capacity of $16 \mathrm{wt} \%$, a record high value reported so far. The stability of the proposed metal-ethylene complexes was tested by extensive calculations such as normal-mode analysis, finite temperature first-principles moleculardynamics (MD) simulations, and reaction path calculations. The phonon and MD simulations indicate that the proposed structures are stable up to $500 \mathrm{~K}$. The reaction path calculations indicate about $1 \mathrm{eV}$ activation barrier for the $\mathrm{TM}_{2}$-ethylene complex to transform into a possible lower energy configuration where the ethylene molecule is dissociated. Importantly, no matter which isometric configuration the $\mathrm{TM}_{2}$-ethylene complex possesses, the TM atoms are able to bind multiple hydrogen molecules with suitable binding energy for room-temperature storage. These results suggest that co-deposition of ethylene with a suitable precursor of $\mathrm{TM}$ or $\mathrm{Li}$ into nanopores of light-weight host materials may be a very promising route to discovering new materials with high-capacity hydrogen absorption properties.
\end{abstract}

DOI: 10.1103/PhysRevB.76.085434

PACS number(s): 68.43.Bc, 81.07.-b, 84.60.Ve

\section{INTRODUCTION}

The success of future hydrogen and fuel-cell technologies is critically dependent upon the discovery of new materials that can store a large amount of hydrogen at ambient conditions. ${ }^{1-3}$ Recently, from quantum-mechanical calculations we found that the $\mathrm{C}=\mathrm{C}$ bond in a single ethylene molecule, similar to $\mathrm{C}_{60}$ and carbon nanotubes, ${ }^{4-8}$ can form a stable complex with transition metals (TMs) such as Ti. ${ }^{9}$ The resulting $\mathrm{TM}_{2}$-ethylene complex attracts up to ten hydrogen molecules via the Dewar-Kubas interaction, ${ }^{10}$ reaching a gravimetric storage capacity of $\sim 14 \mathrm{wt} \% .^{9}$ The interaction between hydrogen molecules and transition metals lies between chemisorption and physisorption, with a binding energy of $\sim 0.4 \mathrm{eV} / \mathrm{H}_{2}$ compatible with room-temperature desorption or absorption at ambient conditions (i.e., at room temperature and under $1 \mathrm{~atm} . \mathrm{H}_{2}$ pressure). ${ }^{3}$

Different from metal decorated $\mathrm{C}_{60}$ or nanotubes, metal$\mathrm{C}_{2} \mathrm{H}_{4}$ complexes are actually existing structures and have been actively studied in the past several decades, with the major goal being to understand the catalytic mechanisms and processes of metals. Experimental spectroscopic data on various complexes, such as $\mathrm{Li}, \mathrm{Mg}, \mathrm{Al}$, and TMs complexed with $\mathrm{C}_{2} \mathrm{H}_{4}$, widely exist in the literature. ${ }^{11-14}$ These complexes were typically synthesized by direct reaction of metal atoms with $\mathrm{C}_{2} \mathrm{H}_{4} / \mathrm{Ar}$ in the gas phase. Early theoretical studies ${ }^{14-18}$ showed that the metal- $\mathrm{C}_{2} \mathrm{H}_{4}$ binding mechanisms could be either electrostatic (e.g., $\mathrm{C}_{2} \mathrm{H}_{4}$-Al), or Dewar-ChattDuncanson bonding (e.g., most $\mathrm{C}_{2} \mathrm{H}_{4} \mathrm{TMs}$ ). The ability of metal- $\mathrm{C}_{2} \mathrm{H}_{4}$ complexes to absorb $\mathrm{H}_{2}$ was realized and investigated in our recent work. ${ }^{9}$
Here we extend our earlier work ${ }^{9}$ and present a detailed theoretical study of the hydrogen absorption on a large number of metal- $\mathrm{C}_{2} \mathrm{H}_{4}$ complexes, including TMs and the alkalimetal Li. We organize the paper as follows. In the next section, we describe the computational methodology. In Sec. III, we discuss $\mathrm{C}_{2} \mathrm{H}_{4} M$ complexes, various isomers of $\mathrm{C}_{2} \mathrm{H}_{4} M_{2}$ complexes, and present the metal binding energies, zerotemperature dynamics of these complexes and their hydrogen absorption properties (including the $\mathrm{H}_{2}$ binding energies and maximum number of $\mathrm{H}_{2}$ that the complex can absorb). In Sec. IV, we discuss the possible reaction paths (i.e., minimum-energy paths) and the activation energies (i.e., barriers) between various isomers of $\mathrm{C}_{2} \mathrm{H}_{4} \mathrm{Ti}_{2}$ complexes. We also discuss an interesting catalytic effect of $\mathrm{Ti}$, similar to the "spillover effect," where a molecularly bound $\mathrm{H}_{2}$ molecule is first dissociated over $\mathrm{Ti}$ and then one of the $\mathrm{H}$ atoms is bonded to carbon, forming a $\mathrm{CH}_{3}$ group. The resulting molecule is isostructural to an "ethanol" molecule and thus called "titanol." The titanol molecule is also able to absorb up to five $\mathrm{H}_{2}$ as molecules with a binding energy of $\sim 0.4 \mathrm{eV} / \mathrm{H}_{2}$ and provide another interesting possibility for high-capacity hydrogen storage materials. In Sec. V, we present high-temperature first-principles molecular-dynamics (MD) studies on selected structures. Due to the small system size, we are able to carry out MD simulations up to $10 \mathrm{ps}$. We show that the proposed complex structures are quite stable and exhibit constructive desorption upon heating without destroying the underlying complex. Our concluding remarks are presented in Sec. VI. 


\section{DETAILS OF CALCULATIONS}

Our first-principles energy calculations were done within density-functional theory using Vanderbilt-type ultra soft pseudopotentials with Perdew-Burke-Ernzerhof exchange correlation, as implemented in the PWSCF package. ${ }^{19}$ We note an unfortunate typographical error in our previous paper ${ }^{9}$ where "Perdew-Zunger" should actually be "Perdew-BurkeErnzerhof." Single molecular complexes have been treated in a supercell of $20 \times 20 \times 20 \AA$ with $\Gamma k$-point and a cutoff energy of $408 \mathrm{eV}$. The structures are optimized until the maximum force allowed on each atom is less than $0.01 \mathrm{eV} / \AA$ for both spin-paired and spin-relaxed cases. The reaction path calculations were carried out using the nudged elastic band (NEB) method. ${ }^{20,21}$ We used a total of 21 images between the reactant and the product, which were fully optimized during the NEB calculations. The MD simulations were carried out within the microcanonical ensemble (NVE) starting with the optimized structure and random initial atom velocities. $^{22,23}$ More details of the MD calculations are given in $\mathrm{V}$.

\section{STRUCTURAL, ELECTRONIC, AND DYNAMICAL PROPERTIES OF $\mathrm{C}_{2} \mathrm{H}_{4} M_{n}$ AND $\mathrm{C}_{2} \mathrm{H}_{4} M_{n}-\mathrm{H}_{x}$ COMPLEXES}

We start by examining various possible configurations of $\mathrm{C}_{2} \mathrm{H}_{4} M_{n}$ complexes and their corresponding $\mathrm{H}_{2}$ absorption properties. We consider both transition metals and light metal $\mathrm{Li}$, and focus on $n=1$ and $n=2$ cases. Complexes with $n>2$ are less attractive for hydrogen storage due to potentially lower capacities and thus are not discussed here and should be avoided in the syntheses.

When one metal atom binds to the ethylene molecule, the configuration shown in Fig. 1(a) is the most energetically favorable one, where the metal atom forms a symmetric bridge "bond" with the $\mathrm{C}=\mathrm{C}$ bond of ethylene. When two metal atoms bind to $\mathrm{C}_{2} \mathrm{H}_{4}$, the complex may adopt several possible configurations. In our initial study, ${ }^{9}$ we focused on the sandwich structure [Fig. 1(b)]. Here we consider two additional isomeric structures: dimer par [Fig. 1(c)] and dimer perp [Fig. 1(e)]. In the sandwich configuration, each $M$ atom is closer to one of the carbon atoms, leading to two different $M-\mathrm{C}$ "bonds." Note that for most transition metals (e.g., Ti), there is no classical chemical covalent bonding between the metal atom and carbon atom. The calculated bond population is found to be nearly zero for these metals. The slight shift of the metal atoms towards different $\mathrm{C}$ atoms only results in a minute contribution of the $M-\mathrm{C}$ covalentlike bond to the overall binding. In just a few cases (e.g., Fe), the metal and carbon atom are bonded more traditionally by a covalent bond, as shown in Fig. 1(d). For this reason, we generally specify these $\mathrm{C}_{2} \mathrm{H}_{4} M_{n}$ structures as "complexes" instead of "molecules."

The binding mechanism of the $\mathrm{C}_{2} \mathrm{H}_{4} T M_{n}$ complex has been discussed in detail in our previous work. ${ }^{9}$ Essentially, the bonding orbital for the TM atoms and $\mathrm{C}_{2} \mathrm{H}_{4}$ results from the hybridization of the lowest-unoccupied molecular orbital (LUMO) of the ethylene molecule and the TM- $d$ orbitals, in accord with Dewar coordination. For Li, the binding mecha-

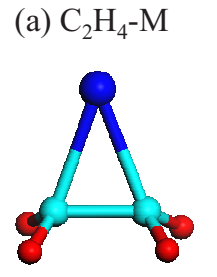

(b) $\mathrm{C}_{2} \mathrm{H}_{4}-\mathrm{M}_{2}$ (sandwich)
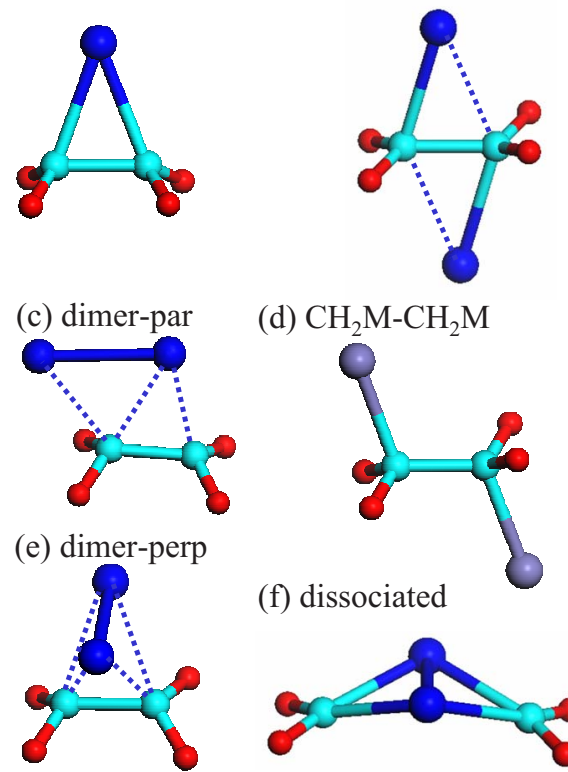

(d) $\mathrm{CH}_{2} \mathrm{M}-\mathrm{CH}_{2} \mathrm{M}$

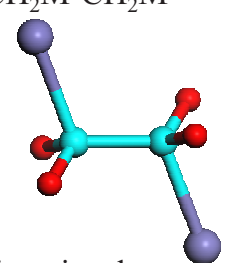

(f) dissociated

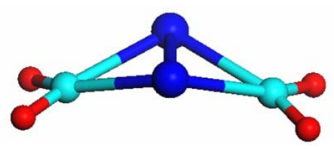

FIG. 1. (Color online) Various configurations of $\mathrm{C}_{2} \mathrm{H}_{4} M_{n}$ (n $=1$ and 2) complexes considered in this study. (a) $\mathrm{C}_{2} \mathrm{H}_{4}$ complexed with one metal atom. (b)-(e) $\mathrm{C}_{2} \mathrm{H}_{4}$ complexed with two metal atoms with different metal binding sites. Note that the bond-stick model is only used for clarity and should not be considered as an implication of the chemical covalent bonding between those atoms. For most metals, there is no classical chemical covalent bonding between the metal and carbon atoms. For a few metals (e.g., Fe), the complexes possess a structure, where $M$ and $\mathrm{C}$ are bonded more traditionally by covalent bonding, as shown in (d). (f) $\mathrm{C}_{2} \mathrm{H}_{4} M_{2}$ complex with dissociated $\mathrm{C}=\mathrm{C}$ bond. Large, medium, and small balls represent $M, \mathrm{C}$, and $\mathrm{H}$ atoms, respectively.

nism is different. In Fig. 2, we show the electronic density of states of the $\mathrm{C}_{2} \mathrm{H}_{4}$ molecule, the $\mathrm{Li}$ atom, and the $\mathrm{C}_{2} \mathrm{H}_{4} \mathrm{Li}$ complex. Projection analysis of the states indicates that the electron in the $2 s$ state of $\mathrm{Li}$ is divided into two halves that are transferred to the LUMO of $\mathrm{C}_{2} \mathrm{H}_{4}$ and the $2 p$ of the $\mathrm{Li}$

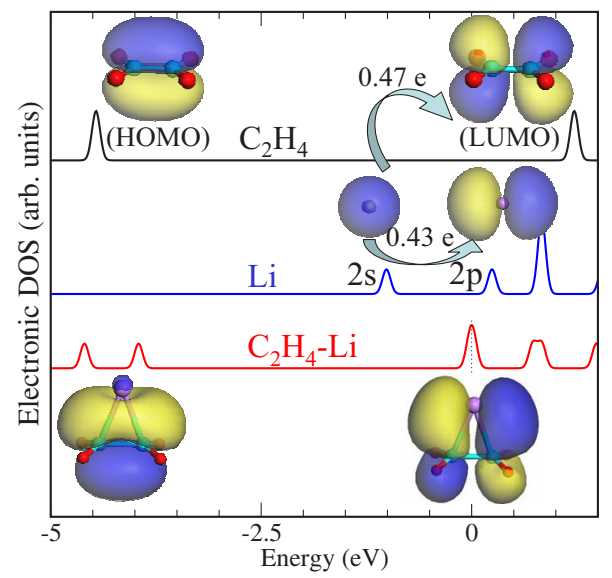

FIG. 2. (Color online) Electronic density of states of $\mathrm{C}_{2} \mathrm{H}_{4}, \mathrm{Li}$ atom, and $\mathrm{C}_{2} \mathrm{H}_{4}+\mathrm{Li}$ complex. The isosurfaces of the relevant molecular orbitals are also shown. The hybridization of the $\mathrm{Li}-2 p$ state and the LUMO of $\mathrm{C}_{2} \mathrm{H}_{4}$ is apparent. See text for further explanation. 
TABLE I. The metal- $\mathrm{C}_{2} \mathrm{H}_{4}$ binding energies (in $\mathrm{eV} / M$ atom) with respect to atomic and bulk energies of various metals, and the average $\mathrm{H}_{2}$ binding energies (in $\mathrm{eV} / \mathrm{H}_{2}$ ) on $\mathrm{C}_{2} \mathrm{H}_{4} M$ for various absorption configurations (see Fig. 3). The maximum number of $\mathrm{H}_{2}$ molecules bonded to each metal is also shown.

\begin{tabular}{|c|c|c|c|c|c|c|c|c|c|c|c|c|c|c|c|c|}
\hline Property/M & $\mathrm{Li}$ & $\mathrm{Sc}$ & $\mathrm{Ti}$ & V & $\mathrm{Cr}$ & $\mathrm{Mn}$ & $\mathrm{Fe}$ & Co & $\mathrm{Ni}$ & $\mathrm{Cu}$ & $\mathrm{Zn}$ & $\mathrm{Zr}$ & Mo & $\mathrm{W}$ & $\mathrm{Pd}$ & $\mathrm{Pt}$ \\
\hline$E_{B}(M$ atomic $)$ & 0.32 & 1.39 & 1.45 & 0.94 & 0.18 & 0.51 & 0.92 & 1.39 & 0.91 & 0.80 & none & 1.91 & 1.02 & 1.71 & 1.95 & 2.52 \\
\hline$E_{B}(M$ bulk $)$ & -1.41 & -2.72 & -3.68 & -4.30 & -3.44 & -3.06 & -1.64 & -2.43 & -1.99 & -2.86 & & -4.23 & -5.19 & -6.65 & -1.86 & -2.82 \\
\hline$E_{B}\left(\right.$ per $\left.\mathrm{H}_{2}\right), M \mathrm{H}_{2}$ & & 0.96 & 1.16 & 1.00 & 0.01 & 0.59 & 1.01 & 0.94 & 1.13 & 0.19 & & 1.90 & 0.85 & 1.80 & 0.83 & 1.33 \\
\hline$E_{B}\left(\right.$ per $\left.\mathrm{H}_{2}\right), M+\mathrm{H}_{2}$ & 0.29 & 0.02 & 0.31 & 0.46 & 0.45 & & & & & & & 0.35 & 0.49 & 0.59 & 0.64 & \\
\hline $\max \mathrm{H}_{2} / M$ & 2 & 5 & 5 & 5 & 5 & 5 & 5 & 3 & 2 & 2 & & 5 & 5 & 5 & 2 & 2 \\
\hline$E_{B}\left(\right.$ per $\left.\mathrm{H}_{2}\right), M \mathrm{H}_{2}+3 \mathrm{H}_{2}$ & & 0.40 & 0.54 & 0.66 & 0.34 & 0.24 & 0.31 & & & & & 0.78 & 0.61 & 0.85 & & \\
\hline$E_{B}\left(\right.$ per $\left.\mathrm{H}_{2}\right), M+5 \mathrm{H}_{2}$ & & 0.28 & 0.46 & 0.53 & 0.21 & 0.18 & 0.34 & & & & & 0.54 & 0.64 & 0.79 & & \\
\hline
\end{tabular}

atom, respectively. Then the $2 p$ orbital of Li and the LUMO of $\mathrm{C}_{2} \mathrm{H}_{4}$ are hybridized for the binding of $\mathrm{Li}$ on the $\mathrm{C}_{2} \mathrm{H}_{4}$. From the isosurfaces of the molecular orbitals (also shown in Fig. 2), it is clear that the molecular orbital of $\mathrm{C}_{2} \mathrm{H}_{4} \mathrm{Li}$ near the zero energy (i.e., the Fermi energy) is a superposition of the LUMO of $\mathrm{C}_{2} \mathrm{H}_{4}$ and the $p$ orbital of the Li atom. Also note that the occupied orbital of the $\mathrm{C}_{2} \mathrm{H}_{4} \mathrm{Li}$ complex at around $-4 \mathrm{eV}$ is about the same as that of the highest occupied molecular orbital (HOMO) of bare $\mathrm{C}_{2} \mathrm{H}_{4}$, except that there is a hole in the upper portion of the orbital due to the $\mathrm{Li}$ ion. The bond analysis does not show any covalent bonding between $\mathrm{C}$ and $\mathrm{Li}$ atoms. For $\mathrm{C}_{2} \mathrm{H}_{4} \mathrm{Li}_{2}$, we observed also a binding mechanism similar to that of $\mathrm{C}_{2} \mathrm{H}_{4} \mathrm{Li}$.

The metal binding energies on ethylene are summarized in Table I and Table II for one metal and two metal complexes, respectively. They are calculated by subtracting the equilibrium total energy $E_{T}$ of the $\mathrm{C}_{2} \mathrm{H}_{4} M_{n}$ complex from the sum of the total energies of free molecular ethylene and of the $M$ atom: $E_{B}(M)=\left[E_{T}\left(\mathrm{C}_{2} \mathrm{H}_{4}\right)+n E_{T}(M)-E_{T}\left(\mathrm{C}_{2} \mathrm{H}_{4} M_{n}\right)\right] / n$. According to the $E_{B}(M$-atomic) values shown in both tables, most TMs that we studied are able to bind relatively strongly to a $\mathrm{C}_{2} \mathrm{H}_{4}$ molecule, except $\mathrm{Cr}$ and $\mathrm{Zn}$. In Table I, the variation of the TM binding energy with the number of TM-3d electrons displays a behavior similar to what was observed previously for the chemisorption of TMs on the surface of a single-walled carbon nanotube. ${ }^{24,25}$ Namely, there exist two energy maxima between a minimum that occurs for the element with five $d$ electrons. Table I also gives the binding energies with respect to bulk metal energies $\left[E_{B}(M\right.$ bulk $\left.)\right]$ while Table II also gives $E_{B}$ with respect to metal dimer energies $\left[E_{B}(M\right.$ dimer $\left.)\right]$. Note that all $E_{B}(M$ bulk $)$ values are negative, indicating endothermic reactions. Apparently, metal atoms in vapor or some metal precursors, instead of bulk metals, are preferred when synthesizing these complex structures.

We next studied the $\mathrm{H}_{2}$ storage capacity of the metalethylene complex, by calculating the interaction between $\mathrm{C}_{2} \mathrm{H}_{4} M_{n}$ and a different number of $\mathrm{H}_{2}$ molecules. We considered various configurations for the hydrogen absorption on a metal center, as shown in Fig. 3. The first $\mathrm{H}_{2}$ molecule absorbed may either be in molecular form [Fig. 3(a)] or in dissociated form [Fig. 3(b)]. For most transition metals, it is possible to absorb more, up to five $\mathrm{H}_{2}$ per $M$ atom. Two of the many possible multiple $\mathrm{H}_{2}$ absorption configurations are shown in Figs. 3(c) and 3(d). For $\mathrm{Li}$, in both $\mathrm{C}_{2} \mathrm{H}_{4} \mathrm{Li}$ and $\mathrm{C}_{2} \mathrm{H}_{4} \mathrm{Li}_{2}$ complexes, each $\mathrm{Li}$ can bind to two $\mathrm{H}_{2}$, resulting in absorption capacity of 10.3 and $16.0 \mathrm{wt} \%$, respectively. The optimized configurations and structural parameters are shown in Fig. 4.

The nature of the metal- $\mathrm{H}_{2}$ interaction is easy to understand. For TMs, since the bonding orbitals are mainly between metal $d$ - and hydrogen $\sigma^{*}$-antibonding orbitals, the mechanism of this interesting interaction can be explained by the Kubas interaction. ${ }^{10}$ For $\mathrm{Li}$, the metal- $\mathrm{H}_{2}$ binding is mainly electrostatic. We summarize the average $\mathrm{H}_{2}$ binding energy for $\mathrm{C}_{2} \mathrm{H}_{4} M$ in Table I. Note that the $\mathrm{H}_{2}$ binding energies for the $\mathrm{C}_{2} \mathrm{H}_{4} M$ complexes differ slightly from those given in our earlier work ${ }^{9}$ for the $\mathrm{C}_{2} \mathrm{H}_{4} M_{2}$ complexes with the sandwich structure, a result of the slightly different electronic structures of the $M$ atoms in the two types of complexes. Nevertheless, in most cases, the $\mathrm{H}_{2}$ binding energies

TABLE II. The metal- $\mathrm{C}_{2} \mathrm{H}_{4}$ binding energies (in eV/M atom) of three isomeric $\mathrm{C}_{2} \mathrm{H}_{4} M_{2}$ configurations (see Fig. 1), with respect to atomic and dimer energies of various metals.

\begin{tabular}{lcccccccccccccccc}
\hline \hline Property/ $M$ & $\mathrm{Li}$ & $\mathrm{Sc}$ & $\mathrm{Ti}$ & $\mathrm{V}$ & $\mathrm{Cr}$ & $\mathrm{Mn}$ & $\mathrm{Fe}$ & $\mathrm{Co}$ & $\mathrm{Ni}$ & $\mathrm{Cu}$ & $\mathrm{Zn}$ & $\mathrm{Zr}$ & $\mathrm{Mo}$ & $\mathrm{W}$ & $\mathrm{Pd}$ & $\mathrm{Pt}$ \\
\hline$E_{B}(M$ atomic), sandwich & 0.69 & 1.39 & 1.47 & 1.21 & 0.05 & 0.37 & 0.83 & 1.30 & 0.70 & 1.41 & none & 1.69 & 0.37 & 1.18 & 1.56 & 1.78 \\
$E_{B}(M$ atomic), dimer par & 0.54 & 1.77 & 2.02 & 1.62 & 0.10 & 0.64 & 1.65 & 1.63 & 1.09 & 1.34 & none & 2.66 & 2.20 & 3.26 & 1.88 & 2.61 \\
$E_{B}(M$ atomic), dimer perp & 0.61 & 1.72 & 2.12 & 1.97 & 0.21 & 0.51 & 1.22 & 1.50 & 1.00 & 1.24 & none & 2.70 & 2.10 & 2.41 & 1.39 & 1.53 \\
$E_{B}(M$ dimer $)$, sandwich & 0.20 & 0.58 & 0.17 & -0.21 & 0.79 & 0.33 & -0.36 & -3.45 & -0.01 & 0.17 & & -0.10 & -1.71 & -1.30 & 0.75 & 0.20 \\
$E_{B}(M$ dimer $)$, dimer par & 0.05 & 0.96 & 0.72 & 0.20 & 0.84 & 0.60 & 0.46 & -3.12 & 0.38 & 0.10 & & 0.87 & 0.12 & 0.78 & 1.08 & 1.03 \\
$E_{B}(M$ dimer $)$, dimer perp & 0.12 & 0.91 & 0.82 & 0.54 & 0.95 & 0.47 & 0.03 & -3.25 & 0.29 & 0.00 & & 0.91 & 0.02 & -0.07 & 0.58 & -0.05 \\
\hline \hline
\end{tabular}




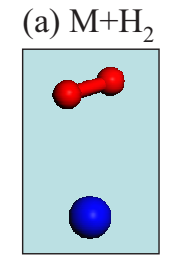

(b) $\mathrm{MH}_{2}$

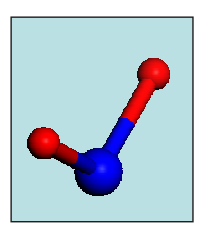

(c) $\mathrm{MH}_{2}+3 \mathrm{H}_{2}$

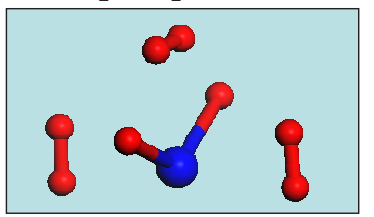

(d) $\mathrm{M}+5 \mathrm{H}_{2}$

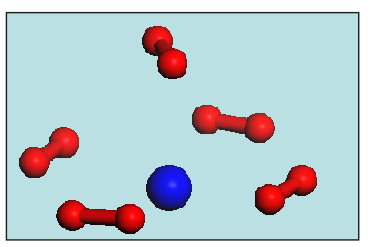

FIG. 3. (Color online) Various configurations that we considered in this study, for the hydrogen absorption on a metal center of a $\mathrm{C}_{2} \mathrm{H}_{4} M_{n}$ complex: (a) one $\mathrm{H}_{2}$ absorbed molecularly; (b) $\mathrm{H}_{2}$ dissociating with two $M-\mathrm{H}$ bond formed; (c) two atomic $\mathrm{H}$ and three $\mathrm{H}_{2}$ molecules; (d) five $\mathrm{H}_{2}$ absorbed as molecules. Large and small balls represent $M$ and $\mathrm{H}$ atoms, respectively.

have the right order of magnitude for room-temperature storage. Since the hydrogens are mainly absorbed molecularly, we also expect fast absorption and desorption kinetics.

In order to test their stability, we further studied the dynamic of the $\mathrm{C}_{2} \mathrm{H}_{4} M_{n}$ complexes by normal-mode analysis. We found no soft (i.e., negative) mode, indicating that the complex structures are stable. Characteristic phonon modes are summarized in Table III, using $\mathrm{Li}$ and $\mathrm{Ti}$ as examples. Our calculated mode frequencies for the $\mathrm{C}_{2} \mathrm{H}_{4}$ molecule agree very well with the experimental values. ${ }^{26}$ Metal binding to $\mathrm{C}_{2} \mathrm{H}_{4}$ elongates and thus softens the $\mathrm{C}=\mathrm{C}$ bond, resulting in lower stretching mode frequencies. Also the softening of the $\mathrm{CH}_{2}$-torsion and $\mathrm{CH}_{2}$-bending modes is obvious. There are three main $M$-related vibrational modes. In two of these modes, $M$ atoms vibrate parallel and perpendicular to the $\mathrm{C}=\mathrm{C}$ bond. In the third mode, metal atoms vibrate perpendicular to the $\mathrm{C}_{2} \mathrm{H}_{4}$ plane. These three modes are unique for the $\mathrm{C}_{2} \mathrm{H}_{4} M_{n}$ complex and therefore should be present in any Raman or IR spectra of a successfully synthesized material.

We also calculated the normal modes of $\mathrm{C}_{2} \mathrm{H}_{4} M_{n}$ complexes absorbed with $\mathrm{H}_{2}$ and did not find any soft modes, indicating that the configurations that we considered indeed correspond to local-energy minima. Among many vibrational

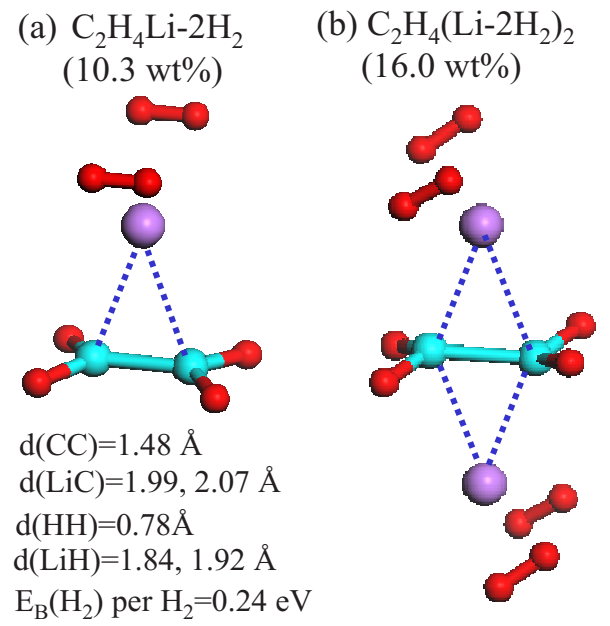

FIG. 4. (Color online) Hydrogen absorption configurations on (a) $\mathrm{C}_{2} \mathrm{H}_{4} \mathrm{Li}$ and (b) $\mathrm{C}_{2} \mathrm{H}_{4} \mathrm{Li}_{2}$ complexes. Note that in both cases, each $\mathrm{Li}$ can bind two $\mathrm{H}_{2}$, resulting in high absorption capacities. Large, medium, and small balls represent $\mathrm{Li}, \mathrm{C}$, and $\mathrm{H}$ atoms, respectively.

modes, we note that the $\mathrm{H}_{2}$ stretching mode is around $330-420 \mathrm{meV}$ for the absorbed $\mathrm{H}_{2}$ molecules, significantly lower than $\sim 540 \mathrm{meV}$ for the free $\mathrm{H}_{2}$ molecule. Such a shift in the mode frequency would be the key feature that can be probed by Raman or IR measurement to confirm a successful synthesis of the structures predicted here. In the lower energy range, there are many $M-\mathrm{H}$ modes that are unique to the complexes. To manifest the $M-\mathrm{H}$ dynamics, we show in Fig. 5 the phonon density of states of $\mathrm{C}_{2} \mathrm{H}_{4} \mathrm{Ti}_{n}-\mathrm{H}_{x}$ complexes weighted by neutron cross sections (note that $\mathrm{H}$ has much larger neutron-scattering cross section than $\mathrm{C}$ and most metals). These plots can provide a useful comparison to experiments when trying to synthesize these materials.

\section{ACTIVATION ENERGIES AND REACTION PATHS BETWEEN DIFFERENT ISOMERS}

The $\mathrm{C}_{2} \mathrm{H}_{4} M_{n}$ and $\mathrm{C}_{2} \mathrm{H}_{4} M_{n}-\mathrm{H}_{x}$ complexes can have several isomeric structures. It is important to know the relative stabilities of these isomers and their implications for the hydrogen absorption properties. We thus studied the activation energies and reaction paths between different isomers of $\mathrm{C}_{2} \mathrm{H}_{4} M_{n}$ complexes. Here we discuss representative results on $M=\mathrm{Ti}$.

TABLE III. Characteristic mode frequencies (meV) for $\mathrm{C}_{2} \mathrm{H}_{4}, \mathrm{C}_{2} \mathrm{H}_{4} \mathrm{Li}_{n}$, and $\mathrm{C}_{2} \mathrm{H}_{4} \mathrm{Ti}_{n}$ complexes. Experimental values for $\mathrm{C}_{2} \mathrm{H}_{4}$ (from Ref. 26) are also shown. Note that the metal- $\mathrm{C}_{2} \mathrm{H}_{4}$ binding significantly softens the $\mathrm{C}=\mathrm{C}$ stretching, $\mathrm{CH}_{2}$-torsion, and $\mathrm{CH}_{2}$-bending modes. The three main $M$ modes give unique signatures for metal- $\mathrm{C}_{2} \mathrm{H}_{4}$ complexes.

\begin{tabular}{lcccccc}
\hline \hline Mode/complex & $\mathrm{C}_{2} \mathrm{H}_{4}$ & $\mathrm{C}_{2} \mathrm{H}_{4}$, expt. & $\mathrm{C}_{2} \mathrm{H}_{4} \mathrm{Li}$ & $\mathrm{C}_{2} \mathrm{H}_{4} \mathrm{Li}_{2}$ & $\mathrm{C}_{2} \mathrm{H}_{4} \mathrm{Ti}$ & $\mathrm{C}_{2} \mathrm{H}_{4} \mathrm{Ti}_{2}$ \\
\hline $\mathrm{C}=\mathrm{C}$ stretching & 202 & 201 & 184 & 172 & 170 & 167 \\
$\mathrm{CH}_{2}$ torsion & 128 & 127 & 99 & 49 & 52 & 56 \\
$\mathrm{CH}_{2}$ bending & $115-165$ & $117-166$ & $84-145$ & $54-141$ & $94-140$ & $73-134$ \\
$M$ vib, $\| \mathrm{C}=\mathrm{C}$ bond & & & 38 & 40 (in phase), 66 (out of phase) & 62 & 15 (in phase), 57 (out of phase) \\
$M$ vib, $\perp \mathrm{C}=\mathrm{C}$ bond & & & 37 & 22 (in phase), 65 (out of phase) & 56 & 22 (in phase), 62 (out of phase) \\
$M$ vib, $\perp \mathrm{C}_{2} \mathrm{H}_{4}$ plane & & 40 & 38 (in phase), 74 (out of phase) & 63 & 29 (in phase), 48 (out of phase) \\
\hline \hline
\end{tabular}




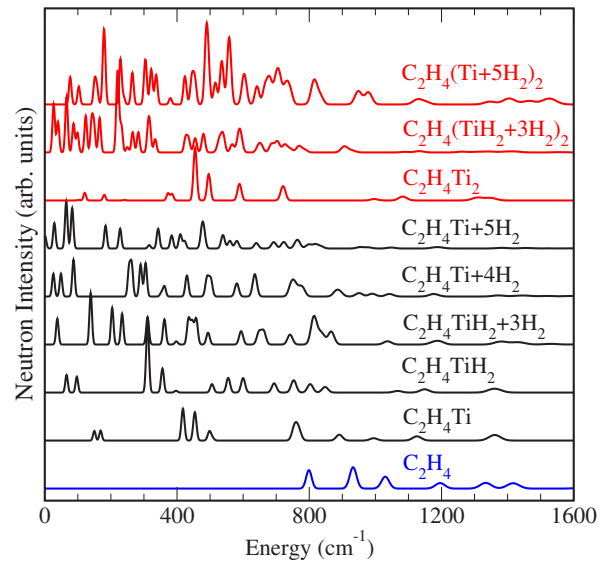

FIG. 5. (Color online) Simulated neutron inelastic spectrum for various $\mathrm{C}_{2} \mathrm{H}_{4} \mathrm{Ti}_{n}-\mathrm{H}_{x}$ configurations. Note that the $M-\mathrm{H}$ dynamics are unique and can be used as a probe to identify these structures. Thus these plots can provide a useful comparison to experiments when trying to synthesize these materials.

We start with the $\mathrm{C}_{2} \mathrm{H}_{4} \mathrm{Ti}+\mathrm{H}_{2}$ complex and consider two possible structural transitions, which lead to lower energy configurations through the dissociation of an $\mathrm{H}_{2}$ molecule over a Ti atom. In the first case, the $\mathrm{H}_{2}$ molecule dissociates on top of the Ti atom. $\mathrm{C}_{2} \mathrm{H}_{4}\left(\mathrm{Ti}+\mathrm{H}_{2}\right)$ and $\mathrm{C}_{2} \mathrm{H}_{4}\left(\mathrm{TiH}_{2}\right)$ are the reactant and product, respectively. Their relaxed structures correspond to the first and last images shown in the top panel of Fig. 6(a). The calculated minimum-energy path for this process gives $\sim 0.25 \mathrm{eV}$ barrier, which is small but still significant since the $\mathrm{C}_{2} \mathrm{H}_{4}\left(\mathrm{Ti}+\mathrm{H}_{2}\right)$ configuration corresponds to a local-energy minimum and possesses a $\mathrm{H}_{2}$ binding energy of $\sim 0.3 \mathrm{eV}$. In the second case, the $\mathrm{H}_{2}$ molecule is first dissociated over $\mathrm{Ti}$ and then one of the $\mathrm{H}$ atoms goes to carbon, forming a $\mathrm{CH}_{3}$ group. The activation energy plot for this process is shown in Fig. 6(b), indicating a very low barrier of only $\sim 0.15 \mathrm{eV}$. Once the product [i.e., the last image of the top panel of Fig. 6(b)] forms, the CCTi-bond angle is very soft, resulting in the zero-temperature structure shown in the inset, which has only $30 \mathrm{meV}$ lower energy than the product. The final structure of the molecule [Fig. 6(b), inset] is isostructural to the "ethanol" molecule and therefore we call it "titanol."

Since the titanol molecule is fairly easy to form, it is important to check if this new complex still possesses the high-capacity $\mathrm{H}_{2}$ absorption property. In Fig. 7, we show several stable hydrogen absorption configurations on a titanol molecule. With only one $\mathrm{H}_{2}$, it can be absorbed molecularly [Fig. 7(a)] with a bind energy of $0.3 \mathrm{eV}$ or absorbed dissociatively [Fig. 7(b)], yielding a $\mathrm{TiH}_{3}$ structure, with a binding energy of about $1.0 \mathrm{eV} / \mathrm{H}_{2}$. We expect that the dissociation process may have a similar barrier to that found in Fig. 6(a). Importantly, the titanol molecule can bind up to five $\mathrm{H}_{2}$ as molecules [Fig. 7(c)] with an average binding energy of $\sim 0.4 \mathrm{eV} / \mathrm{H}_{2}$.

Next, we study the $\mathrm{C}_{2} \mathrm{H}_{4} M_{2}$ dimer structures. For Ti, the dimer-perp structure [Fig. 8(a)] has lower total energy than the isomeric sandwich structure [Fig. 1(b)] and dimer-par structure [Fig. 1(c)]. Figure 8(c) shows the activation barrier for the transition from the sandwich configuration to the
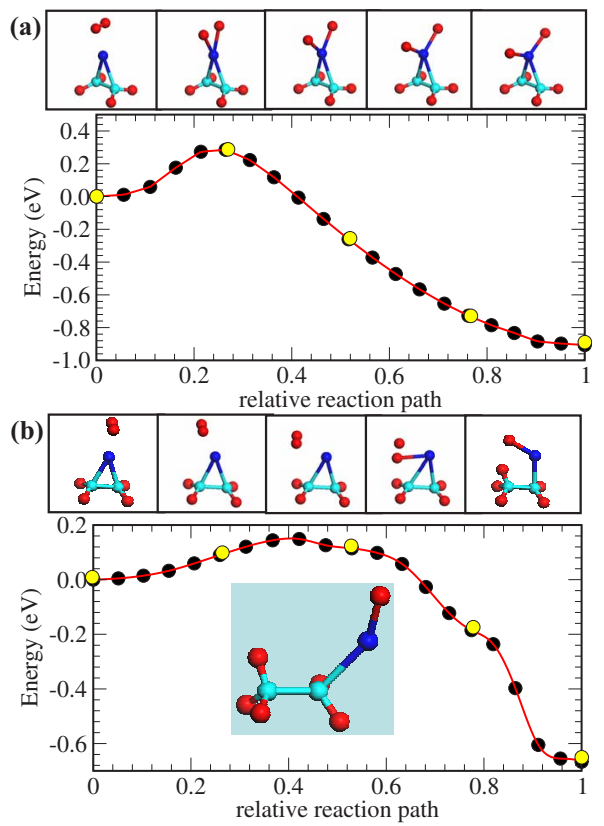

FIG. 6. (Color online) (a) The minimum-energy path for the dissociation of the $\mathrm{H}_{2}$ molecule over the Ti atom complexed with $\mathrm{C}_{2} \mathrm{H}_{4}$. An energy barrier of $\approx 0.25 \mathrm{eV}$ is found for the dissociation. A total of 21 images were used in the NEB calculations, five of which are shown on the top. Marked circles in the potential plot are the points corresponding to these five images. (b) The activation energy plot for the formation of titanol-molecule from $\mathrm{C}_{2} \mathrm{H}_{4} \mathrm{Ti}$ $+\mathrm{H}_{2}$ complex, indicating a very low barrier of $\approx 0.15 \mathrm{eV}$. Once the final product forms, the CCTi-bond angle is very soft, resulting in the zero-temperature structure shown in the inset.

dimer-perp configuration. The activation energy is about $0.55 \mathrm{eV}$. Shown in Fig. 8(b) is one of the stable configurations that we identified for the hydrogen absorption on the $\mathrm{C}_{2} \mathrm{H}_{4} \mathrm{Ti}_{2}$ dimer-perp structure. Apparently, regardless which isomer of $\mathrm{C}_{2} \mathrm{H}_{4} \mathrm{Ti}_{2}$ that we have, the complex is always able to bind multiple hydrogen molecules.

Finally, one may ask whether it is possible for the metal to catalyze and dissociate the $\mathrm{C}_{2} \mathrm{H}_{4}$ molecule (i.e., break the $\mathrm{C}=\mathrm{C}$ bond), forming a more stable structure as shown in Fig. 1(f). Our calculations show that the activation energies for a sandwich to dissociated $\mathrm{C}_{2} \mathrm{H}_{4}$ [Fig. 9(a)] and a dimerperp to dissociated $\mathrm{C}_{2} \mathrm{H}_{4}$ configurations [Fig. 9(b)] are both large, $\sim 1.1 \mathrm{eV}$. Thus it is very unlikely that the dissociation would happen under near ambient conditions. Interestingly, we found that even the dissociated structure can still absorb multiple $\mathrm{H}_{2}$, in which case, the system is somewhat similar to a Ti metallocarbohedryne (met-car) cluster. ${ }^{27,28}$

\section{FINITE-TEMPERATURE FIRST-PRINCIPLES MD SIMULATIONS}

In order to further test the stability of the $\mathrm{C}_{2} \mathrm{H}_{4} M_{n}-\mathrm{H}_{x}$ complexes and the relative strength of different interactions (such as $M-\mathrm{C}_{2} \mathrm{H}_{4} M-\mathrm{H}_{2}$ interactions) and to identify possible reaction paths, we have carried out extensive first-principles MD simulations in the microcanonical ensemble (NVE). ${ }^{22,23}$ We emphasize that our purpose was not to obtain the desorp- 

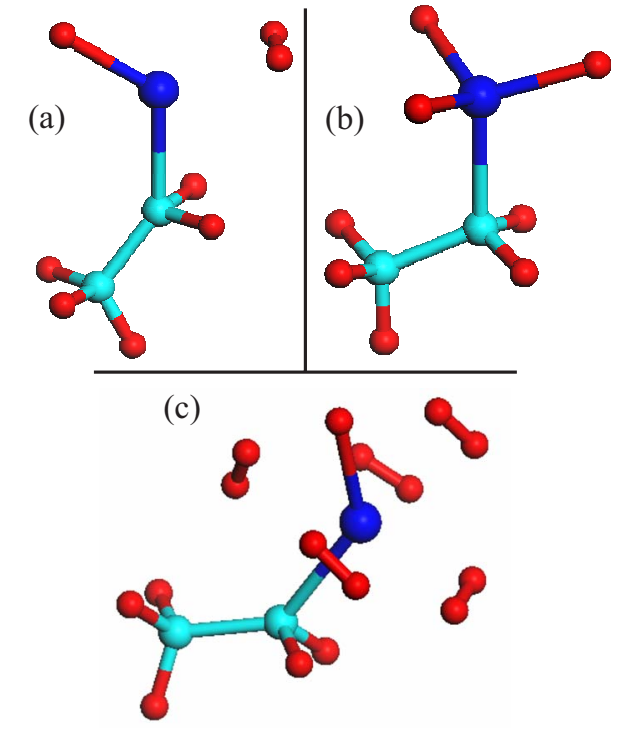

FIG. 7. (Color online) Hydrogen absorption on a titanol molecule. (a) One $\mathrm{H}_{2}$ binds molecularly to Ti, with a binding energy of $0.3 \mathrm{eV}$. (b) One $\mathrm{H}_{2}$ is dissociated, yielding $\mathrm{TiH}_{3}$ structure. The corresponding binding energy is about $1.0 \mathrm{eV} / \mathrm{H}_{2}$. (c) Five $\mathrm{H}_{2}$ bind as molecules to the titanol molecule with an average binding energy of $0.4 \mathrm{eV} / \mathrm{H}_{2}$. Large, medium, and small balls represent Ti, C, and $\mathrm{H}$ atoms, respectively.

tion temperature from the MD simulations, but rather to make sure that we are not missing other stable phases and to show that $\mathrm{H}_{2}$-desorption can occur without destroying the underlying $M-\mathrm{C}_{2} \mathrm{H}_{4}$ complex.

The system is first optimized and then random initial velocities are generated to yield twice the target temperature.

(a)
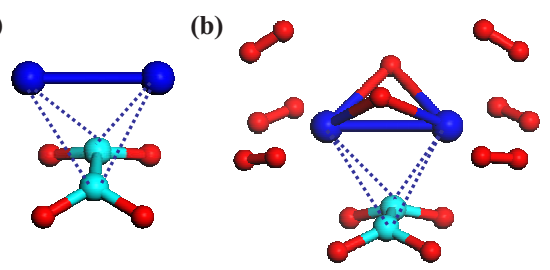

(c)

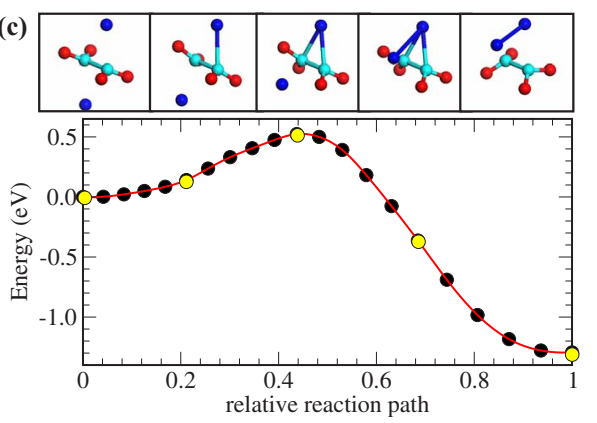

FIG. 8. (Color online) (a) Bare $\mathrm{C}_{2} \mathrm{H}_{4} \mathrm{Ti}_{2}$ dimer-perp complex. (b) The complex with seven $\mathrm{H}_{2}$ absorbed. Note that there also exists other stable configurations that not discussed here. (c) The minimum-energy path for the transition from $\mathrm{C}_{2} \mathrm{H}_{4} \mathrm{Ti}_{2}$ sandwich configuration to dimer-perp configuration. The activation energy is about $0.55 \mathrm{eV}$. Note that regardless of which isomer of $\mathrm{C}_{2} \mathrm{H}_{4} \mathrm{Ti}_{2}$ we have, the resulting complex is able to bind multiple hydrogen molecules.
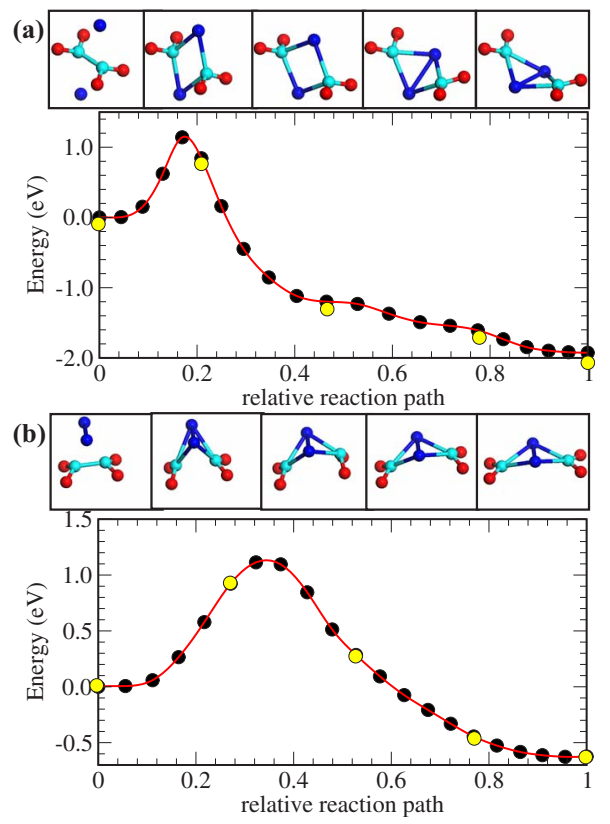

FIG. 9. (Color online) The minimum-energy paths for the transitions (a) from the $\mathrm{C}_{2} \mathrm{H}_{4} \mathrm{Ti}_{2}$ sandwich to the dissociated configuration and (b) from $\mathrm{C}_{2} \mathrm{H}_{4} \mathrm{Ti}_{2}$ dimer perp to the dissociated $\mathrm{C}_{2} \mathrm{H}_{4}$ configuration, respectively. In both cases, there are large energy barriers on the order of $1.1 \mathrm{eV}$.

When the system is in equilibrium, half of this energy goes to the potential and therefore the final temperature oscillates around the target temperature. We note that due to the small atomic mass of some elements (e.g., Li and $\mathrm{H}$ ) in our system, it is essential to use a small MD time step such as $0.5 \mathrm{fs}$. Furthermore, convergence criteria for energy at each MD iteration should be very accurate (we used $10^{-7} \mathrm{eV}$ ) in order to avoid total energy/temperature drift (i.e., change in the total energy/temperature as a function of simulation time). Since we are studying an isolated molecular complex in free space, it is also important that we eliminate the six degrees of freedom (i.e., three rotations and three translations) of the molecule. When this is not done, we observed that the input temperature goes to totally uniform translation or/rotation of the molecules rather than populating the vibrational modes after 1-2-ps simulations. In our simulations, we fixed one of the carbon atoms and then two components of position of the other carbon atom and one component of $M$ atom position (which prevents the rotation of the molecule in the CCM plane). In this way, the total degrees of freedom allowed in our simulation are $N_{F}=3 \times(N-6)$, as expected for an isolated molecule. The temperature of the system is defined as $T(t)=\sum_{i} m_{i} v_{i}^{2} /\left(2 k_{B} N_{F}\right)$, where $i$ runs over the atoms of the complex and $k_{B}$ is Boltzmann's constant. The relative fluctuation is of the order of $1 / \sqrt{N_{F}}$. We also note that since our system is very small (i.e., about a dozen atoms), it is basically a collection of a small number of harmonic oscillators and therefore temperature fluctuations are large. In fact, trying to control system temperature through velocity scaling $^{22,23}$ at a small time interval does not work and yields wrong results. The microcanonical ensemble is thus the best for our purpose and as we shall see below it works well 


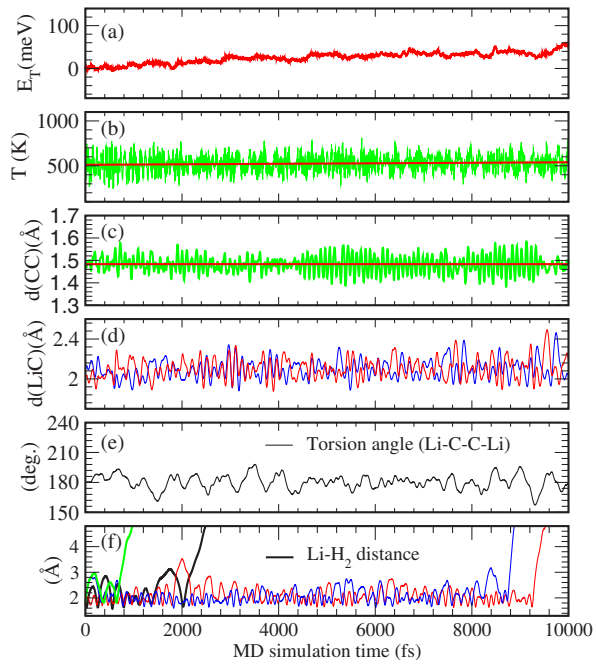

FIG. 10. (Color online) First-principles MD results at $500 \mathrm{~K}$ for the $\mathrm{C}_{2} \mathrm{H}_{4} \mathrm{Li}_{2}+4 \mathrm{H}_{2}$ complex. Shown are the time evolution of various quantities, including total energy (a) and temperature (b) of the system, C-C (c) and Li-C (d) bond distances, and Li-C-C-Li torsion angle (e). The bottom panel (f) shows the distance between the $\mathrm{Li}$ atom and the hydrogen center of mass, indicating successive desorption of $\mathrm{H}_{2}$ molecules along the simulation.

provided that a small time step is used and the total energy/ force calculations are accurate enough. Here we present representative results on $M=\mathrm{Li}, \mathrm{Ti}$ as examples.

Our MD results for $\mathrm{C}_{2} \mathrm{H}_{4} \mathrm{Li}_{2}+4 \mathrm{H}_{2}$ at $500 \mathrm{~K}$ are summarized in Fig. 10. The constant of motion plot shows only $50-\mathrm{meV}$ drift in total energy over 10 -ps simulation time, which causes a small temperature drift. The C-C and Li-C distances, shown in Figs. 10(c) and 10(d) respectively, indicate that the bare $\mathrm{C}_{2} \mathrm{H}_{4} \mathrm{Li}_{2}$ molecule is stable at this temperature. The torsion angle $\mathrm{Li}-\mathrm{C}-\mathrm{C}$-Li shows no sign of Li-dimer formation and oscillates around $180^{\circ}$. The bottom panel in Fig. 10 shows the distance between $\mathrm{Li}$ atoms and the center of mass of $\mathrm{H}_{2}$ molecules, indicating the successive release of hydrogen molecules from the system. The first $\mathrm{H}_{2}$ leaves the system around 400 fs. The fluctuations in the distances become very large at $2000 \mathrm{fs}$, resulting from the release of another hydrogen molecule. Around 8-10 ps, the other two hydrogen molecules also leave the system. Even though with 10 -ps MD simulations, it is not possible to get reliable temperatures; the results are still very promising and suggest that the $\mathrm{C}_{2} \mathrm{H}_{4} \mathrm{Li}_{2}$ system can stay intact at $500 \mathrm{~K}$ while it releases four hydrogen molecules.

We next studied the stability of the $\mathrm{C}_{2} \mathrm{H}_{4} \mathrm{Ti}_{n}$ system. We performed $\mathrm{MD}$ simulations up to $10 \mathrm{ps}$ on $\mathrm{C}_{2} \mathrm{H}_{4} \mathrm{Ti}_{2}$ (sandwich), $\mathrm{C}_{2} \mathrm{H}_{4} \mathrm{Ti}+\mathrm{H}_{2}, \mathrm{C}_{2} \mathrm{H}_{4}\left(\mathrm{Ti}+5 \mathrm{H}_{2}\right)_{2}$ (sandwich), and the titanol molecule $\left(\mathrm{CH}_{3} \mathrm{CH}_{2} \mathrm{TiH}\right)$ at 300 and $500 \mathrm{~K}$. In the simulations on the two metal sandwich systems, we did not observe any Ti-dimer formation. In the case for $\mathrm{C}_{2} \mathrm{H}_{4} \mathrm{Ti}$ $+\mathrm{H}_{2}$, we did observe the spill-over effect, where the $\mathrm{H}_{2}$ is dissociated over $\mathrm{Ti}$ and $\mathrm{C}$ and then Ti moved away with one hydrogen atom attached to it. This is essentially the titanol formation process that we discussed in the previous section.

The MD results for the $\mathrm{C}_{2} \mathrm{H}_{4}\left(\mathrm{Ti}+5 \mathrm{H}_{2}\right)_{2}$ system at $500 \mathrm{~K}$ are summarized in Fig. 11. During the 10-ps simulation time,

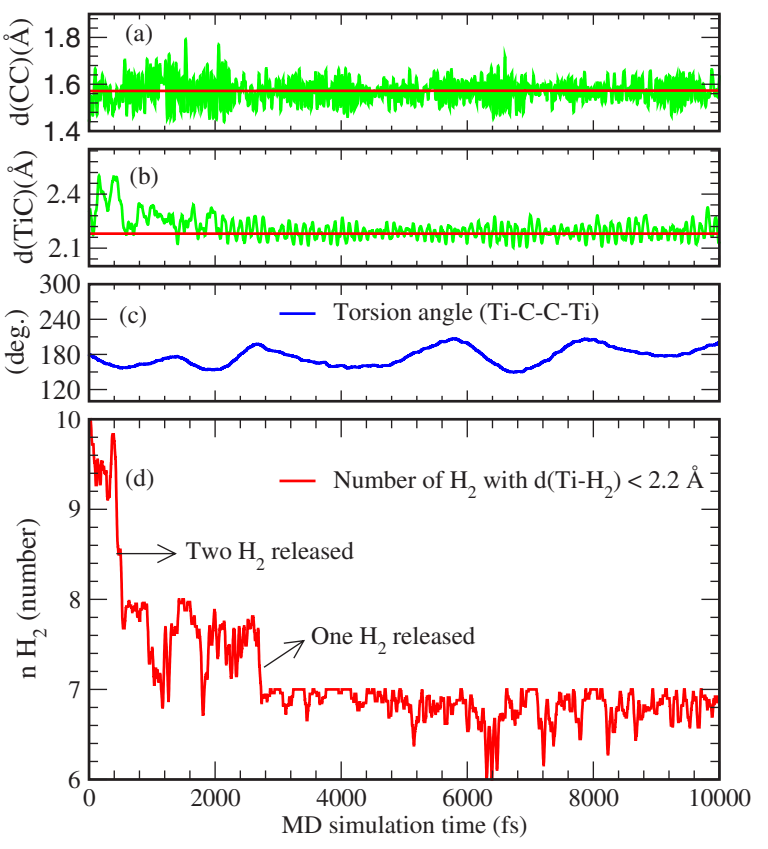

FIG. 11. (Color online) First-principles MD results at $500 \mathrm{~K}$ for the $\mathrm{C}_{2} \mathrm{H}_{4}\left(\mathrm{Ti}+5 \mathrm{H}_{2}\right)_{2}$ sandwich complex. Various quantities are shown, including C-C (a) and Ti-C (b) bond distances, and Ti-C$\mathrm{C}$-Ti torsion angle (c). The bottom panel (d) shows the number of $\mathrm{H}_{2}$ molecules that are within $2.2 \AA$ of Ti atoms. It indicates successive desorption of $\mathrm{H}_{2}$ molecules in the course of the simulation.

both $\mathrm{C}-\mathrm{C}$ and $\mathrm{Ti}-\mathrm{C}$ bond distances oscillate around their equilibrium lengths without any indication of instability. Similarly, the Ti-C-C-Ti torsion angle also slowly oscillates around its equilibrium value of $180^{\circ}$ and does not show any evidence for $\mathrm{Ti}-\mathrm{Ti}$ dimer formation for which the torsion angle is supposed to be about $57^{\circ}$. Figure $11(\mathrm{~d})$ shows the number of $\mathrm{H}_{2}$ molecules that are close to a Ti atom (within a 2.2- $\AA$ distance), showing that initially two $\mathrm{H}_{2}$ molecules are released and then another $\mathrm{H}_{2}$ molecule is released at around 2.4 ps. Above $6 \mathrm{ps}$, the number of $\mathrm{H}_{2}$ fluctuates indicating that the distances are going beyond $2.2 \AA$ more often. Probably if we had run the MD simulation further, we would lose the remaining $\mathrm{H}_{2}$ molecules that are attached to the $\mathrm{Ti}$ atoms.

As a final example, in Fig. 12, we present results from a 10 -ps $\mathrm{MD}$ run on titanol $+5 \mathrm{H}_{2}$ molecules at $500 \mathrm{~K}$. The $\mathrm{C}-\mathrm{C}$ and Ti-C distances indicate that the bare titanol molecule is stable at this temperature. The $\mathrm{C}-\mathrm{C}$-Ti angle shown in Fig. 12(c) indicates that the $\mathrm{C}-\mathrm{C}$-Ti bond angle is very soft, exhibiting large amplitude motion. Around $5 \mathrm{ps,} \mathrm{Ti} \mathrm{actually}$ goes to the middle of two carbon atoms, returning to our original $\mathrm{C}_{2} \mathrm{H}_{4}$ Ti-like configuration. As we discussed in the previous section, these two configurations are almost degenerate. The last panel shows the number of $\mathrm{H}$ atoms that are within $2.2 \AA$ of the Ti atom. Three successive constructive desorptions of $\mathrm{H}_{2}$ molecule are evident.

In summary, our MD results discussed above on different systems indicate that the sandwich configuration of $\mathrm{C}_{2} \mathrm{H}_{4} \mathrm{Ti}_{2}$ is quite stable and can bind $\mathrm{H}_{2}$ molecules and then release them at elevated temperature. Similarly, $\mathrm{C}_{2} \mathrm{H}_{4} \mathrm{Li}_{2} \mathrm{MD}$ results also suggest that $\mathrm{Li}$ is another promising option even though the strength of the interactions is at the low side. Finally, 


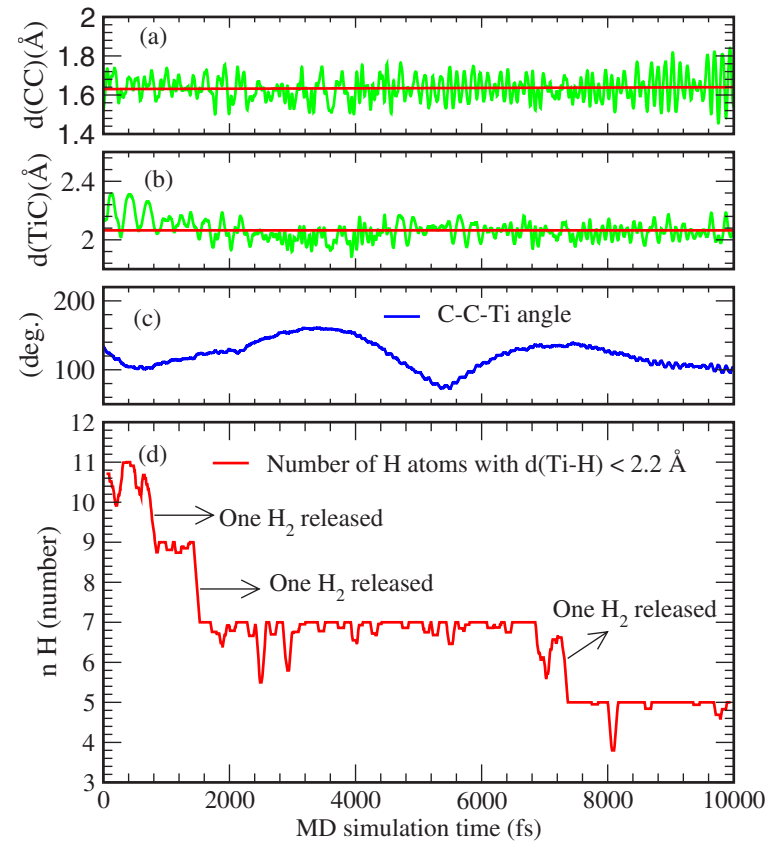

FIG. 12. (Color online) Various quantities (same as in Fig. 11) obtained from MD simulations of the titanol $+5 \mathrm{H}_{2}$ system at $500 \mathrm{~K}$.

thanks to MD simulations, we discovered a new configuration, titanol, which is derived from the $\mathrm{C}_{2} \mathrm{H}_{4} \mathrm{Ti}+\mathrm{H}_{2}$ system and capable of binding five $\mathrm{H}_{2}$ molecules and then releasing them at high temperature without breaking down its structure.

\section{CONCLUSIONS}

Our conclusions are summarized as follows:

(i) We showed that the $\mathrm{C}=\mathrm{C}$ bond in ethylene can mimic the double bond in other carbon structures like $\mathrm{C}_{60}$, in terms of binding metal atoms and the hydrogen absorption properties. The small system size of the $M$-ethylene complex allowed us to do very detailed studies such as long MD simulations and reaction path calculations, which were very difficult to perform otherwise. Most of the results that we found, such as $\mathrm{H}_{2}$ dissociation and titanol formation, should be valid for other Ti-decorated nanostructures.

(ii) For light transition metals, we showed that the initial $\mathrm{H}_{2}$ absorption could be either molecular with binding energy of $\sim 0.3 \mathrm{eV}$ or it could be chemical by $\mathrm{TiH}_{2}$ formation with a binding energy of $\sim 1.0-1.5 \mathrm{eV}$. However, there is a barrier of $\sim 0.25 \mathrm{eV}$ for this process. Since the molecular $\mathrm{H}_{2}$ has a binding energy of $\sim 0.3 \mathrm{eV}$, the dissociation could not be observed. Indeed, in our MD simulations, we did not see conversion from $\mathrm{Ti}+\mathrm{H}_{2}$ to $\mathrm{TiH}_{2}$. Instead, we discovered that there is a very-low-energy barrier for the simultaneous dissociation of $\mathrm{H}_{2}$ and formation of $\mathrm{CH}$ bonding (similar to spill-over effect) through the $\mathrm{Ti}$ atom. For the case of $\mathrm{C}_{2} \mathrm{H}_{4} \mathrm{Ti}+\mathrm{H}_{2}$, this reaction yielded a new molecule which is isostructural to ethanol and can bind five hydrogen molecules with an average binding energy of $\sim 0.4 \mathrm{eV}$.

(iii) We showed that the sandwich configuration of $\mathrm{C}_{2} \mathrm{H}_{4} M_{2}$ is quite stable for both transition metals and Li. There are high-energy barriers for the transition to dimer configurations. Our 10-ps MD simulations did not show any evidence for dimerization.

(iv) From our results, it is clear that $\mathrm{C}_{2} \mathrm{H}_{4} M_{n}$ system could have a very rich phase diagram with different configurations. However, for all the isomer configurations that we have investigated, the complex is always able to bind multiple hydrogen molecules with high absorption capacity. Hence these results suggest that co-deposition of transition/lithium metals with small organic molecules into nanopores of low-density materials could be a very promising direction for discovering new materials with better storage properties.

(v) We note that there are many existing experimental studies of small organic molecules with transition metals in gas phase by mass spectroscopy. In these experiments, the metal atoms are obtained by laser evaporation of bulk metal and then condensed with mixture of Ar and ethylene (or benzene) gas onto a cold substrate. In this way, it was possible to trap $M_{x}\left(\mathrm{C}_{2} \mathrm{H}_{4}\right)_{y}$ types of complexes in an argon matrix and do spectroscopic experiments on them. We hope that our study will reenergize these studies with the focus on hydrogen absorption properties of these systems. It may be possible to use $\mathrm{H}_{2}$ rather than Ar to prepare these clusters in a $\mathrm{H}_{2}$ matrix. Such studies would be very important as a proof of concept and that should be the current emphasis.

\section{ACKNOWLEDGMENTS}

We acknowledge partial DOE support from EERE Grant No. DE-FC36-04GO14282 (W.Z., T.Y.) and BES Grant No. DE-FG02-98ER45701 (S.C.). S.C. and E.D. acknowledge partial support from TÜBİTAK under Grant No. TBAG104T536. We thank J. Curtis and R. Cappelletti for fruitful discussions.

\footnotetext{
*Electronic address: taner@nist.gov

${ }^{1}$ R. Coontz and B Hanson, in Towards a Hydrogen Economy, special issue of Science 305, 957 (2004).

${ }^{2}$ G. W. Crabtree, M. S. Dresselhaus, and M. V. Buchanan, Phys. Today 57, 39 (2004).

${ }^{3}$ A. Zuttel, Mater. Today 6, 24 (2003).

${ }^{4}$ T. Yildirim and S. Ciraci, Phys. Rev. Lett. 94, 175501 (2005).

${ }^{5}$ T. Yildirim, J. Iniguez, and S. Ciraci, Phys. Rev. B 72, 153403
}

(2005).

${ }^{6}$ Y. Zhao, Y.-H. Kim, A. C. Dillon, M. J. Heben, and S. B. Zhang, Phys. Rev. Lett. 94, 155504 (2005).

${ }^{7}$ S. Dag, Y. Ozturk, S. Ciraci, and T. Yildirim, Phys. Rev. B 72, 155404 (2005).

${ }^{8}$ B. Kiran, A. K. Kandalam, and P. Jena, J. Chem. Phys. 124, 224703 (2006).

${ }^{9}$ E. Durgun, S. Ciraci, W. Zhou, and T. Yildirim, Phys. Rev. Lett. 
97, 226102 (2006).

${ }^{10}$ Metal Dihydrogen and Bond Complexes-Structure, Theory and Reactivity, edited by G. J. Kubas (Kluwer Academic/Plenum, New York, 2001).

${ }^{11}$ L. Manceron and L. Andrews, J. Phys. Chem. 90, 4514 (1986).

${ }^{12}$ J. Chen, T. H. Wong, Y. C. Cheng, K. Montgomery, and P. D. Kleiber, J. Chem. Phys. 108, 3909 (1998).

${ }^{13}$ L. Manceron and L. Andrews, J. Phys. Chem. 93, 2964 (1989).

${ }^{14}$ G. A. Ozin, W. J. Power, T. H. Upton, and W. A. Goddard III, J. Am. Chem. Soc. 100, 4750 (1978).

${ }^{15}$ M. E. Alikhani and Y. Bouteiller, J. Phys. Chem. 100, 16092 (1996).

${ }^{16}$ M. Sodupe, C. W. Bauschlicher, S. R. langhoff, and H. Partridge, J. Phys. Chem. 96, 2118 (1992).

${ }^{17}$ M. R. A. Blomberg, P. E. M. Siegbahn, and M. Svensson, J. Phys. Chem. 96, 9794 (1992).

${ }^{18}$ I. Papai, J. Mink, R. Fournier, and D. R. Salahub, J. Phys. Chem. 97, 9986 (1993).
${ }^{19}$ S. Baroni, A. Dal Corso, S. de Gironcoli, and P. Giannozzi, http:// www.pwscf.org

${ }^{20}$ G. Mills and H. Jonsson, Phys. Rev. Lett. 72, 1124 (1994).

${ }^{21}$ G. Henkelman and H. Jansson, J. Chem. Phys. 133, 9978 (2000).

${ }^{22}$ D. Marx and J. Hutter, in Modern Methods and Algorithms of Quantum Chemistry, edited by J. Grotendorst (NIC, FZ Julich, 2000), pp. 301-449.

${ }^{23}$ D. Frenkel and B. Smith, Understanding Molecular Simulation (Acaemic Press, New York, 1996).

${ }^{24}$ E. Durgun, S. Dag, V. M. K. Bagci, O. Gülseren, T. Yildirim, and S. Ciraci, Phys. Rev. B 67, 201401(R) (2003).

${ }^{25}$ E. Durgun, S. Dag, S. Ciraci, and O. Gülseren, J. Phys. Chem. B 108, 575 (2004).

${ }^{26}$ R. Georges, M. Bach, and M. Herman, Mol. Phys. 97, 279 (1999).

${ }^{27}$ Y. Zhao, A. C. Dillon, Y.-H. Kim, M. J. Heben, and S. B. Zhang, Chem. Phys. Lett. 425, 273 (2006).

${ }^{28}$ N. Akman, E. Durgun, T. Yildirim, and S. Ciraci, J. Phys.: Condens. Matter 18, 9509 (2006). 